\title{
Associação entre padrões alimentares com fatores socioeconômicos e ambiente alimentar em uma cidade do Sul do Brasil
}

\author{
Association between dietary patterns and socioeconomic factors \\ and food environment in a city in the South of Brazil
}

Caroline Marques de Lima Cunha (https://orcid.org/0000-0002-0529-1498) ${ }^{1}$

Raquel Canuto (https://orcid.org/0000-0002-4042-1913) ${ }^{1}$

Priscila Bárbara Zanini Rosa (https://orcid.org/0000-0002-1774-073X) ${ }^{1}$

Luana Schimmelpfennig Longarai (http://orcid.org/0000-0002-9692-7286) ${ }^{2}$

Ilaine Schuch (https://orcid.org/0000-0001-6861-4365) ${ }^{1}$
${ }^{1}$ Programa de PósGraduação em Alimentação, Nutrição e Saúde,

Departamento de Nutrição, Universidade Federal do Rio Grande do Sul. R. Ramiro Barcelos 2.400, Santa Cecília. 90035-003. Porto Alegre RS Brasil. ischuch@hcpa.edu.br ${ }^{2}$ Curso de Graduação em Nutrição, Universidade Federal do Rio Grande do Sul. Porto Alegre RS Brasil.

\begin{abstract}
The aim of this study was to investigate the association between dietary patterns and demographic and socioeconomic factors and the food environment among adults and older persons in a city in the south of Brazil. We conducted a cross-sectional study with people of both sexes aged between 20 and 70 years. Dietary patterns were identified using principal component analysis. Poisson regression was used to estimate crude and adjusted prevalence ratios and $95 \%$ confidence intervals. Four dietary patterns were identified: Healthy; Traditional; Refined Carbs and Sugars; and Fast Food. Positive associations were found between being female and higher income and the Healthy dietary pattern; being black or brown and living in a household with at least six members and the Traditional and Refined Carbs and Sugars patterns; and higher education and the fast-food dietary pattern. Having main meals at home was associated with the Traditional pattern and having lunch or dinner away from home was the associated with Refined Carbs and Sugars and fast-food patterns. Lower socioeconomic status leads to higher consumption of the Traditional and/or Refined Carbs and Sugars dietary patterns, while higher socioeconomic status appears to enable individuals to choose between healthy or fast-food patterns.
\end{abstract}

Key words Food patterns, Socioeconomic factors, Socio-spatial health inequalities
Resumo Objetivou-se estudar a associação entre padrão alimentar, fatores demográficos, socioeconômicos e o ambiente alimentar em uma população de adultos e idosos de uma cidade do Sul do Brasil. Estudo transversal com pessoas de ambos os sexos e idade entre 20 e 70 anos. Os padrões alimentares foram identificados por meio da análise de componentes principais. A regressão de Poisson foi utilizada para estimar as razões de prevalência brutas e ajustadas e os intervalos de $95 \%$ de confiança (IC 95\%). Quatro padrões alimentares foram identificados: saudável, tradicional, carboidratos e açúcares refinados e fast food. Encontraram-se associações positivas entre: sexo feminino e maior renda com o padrão alimentar saudável; cor da pele preta ou parda e domicílios com seis ou mais moradores com padrões alimentares tradicional e carboidratos e açúcares refinados; maior escolaridade com o padrão fast food. Realizar as principais refeições em casa associou-se ao padrão tradicional, e almoço ou jantar fora do domicílio com os padrões carboidratos e açúcares refinadose fast food. Posição socioeconômica mais baixa leva ao maior consumo do padrão alimentar tradicional elou rico em carboidratos e açúcares, e uma posição socioeconômica mais elevada parece permitir uma escolha entre padrão saudável ou fast food.

Palavras-chave Padrões alimentares, Fatores socioeconômicos, Desigualdades socioespaciais em saúde 


\section{Introdução}

Os padrões alimentares representam um retrato geral do consumo de alimentos e de nutrientes, $\mathrm{e}$ são caracterizados com base no hábito de ingestão usual ${ }^{1}$. Nas últimas décadas, a estrutura dos padrões alimentares em diversos países, inclusive no Brasil, vem sofrendo modificações importantes, principalmente pelo aumento na ingestão de gorduras, açúcares e alimentos ultraprocessados e pela redução no consumo de alimentos considerados de alto valor nutricional, como frutas e hortaliças. E tais alterações estão associadas a doenças crônicas não transmissíveis (DCNT) ${ }^{2,3}$.

Estudos internacionais e brasileiros mostram associações consistentes entre padrão alimentar e características sociais, econômicas e de estilo de vida, e apresentam outros fatores que também podem interferir nos padrões de consumo alimentar, como tamanho do domicílio, estado civil e cor da pele do indivíduo ${ }^{4-7}$.

Mais recentemente, o ambiente alimentar vem sendo apontado como uma importante característica social na determinação do consumo alimentar dos indivíduos. O ambiente alimentar é definido a partir de quatro dimensões, incluindo os meios físico, econômico, político e sociocultural. Essas dimensões se referem às características de disponibilidade, qualidade e promoção, ao custo, às regulamentações e normatizações e aos aspectos socioculturais, que incluem normas, representações e significados do alimento para um indivíduo ou grupo ${ }^{8}$.

$\mathrm{O}$ ambiente alimentar influencia os padrões alimentares por meio da disponibilidade, do acesso, do preço e da qualidade dos alimentos, e também por fatores individuais como cultura, preferência, aceitabilidade e conhecimento do indivíduo em relação ao alimento ${ }^{9,10}$. A distância entre os pontos de comércio e a residência, assim como a forma de deslocamento para a compra dos itens alimentares, são, portanto, fatores que constituem o ambiente alimentar.

Características socioeconômicas e ambientais não se distribuem geograficamente de forma uniforme nas grandes cidades. Populações que vivem em áreas de baixa renda e alta vulnerabilidade social, como as favelas nas grandes cidades, compartilham características sociodemográficas e ambientes sociais diferentes daqueles dos indivíduos que vivem em áreas de média e alta renda ${ }^{11}$ e podem ser mais vulneráveis a padrões alimentares menos saudáveis.

Estudos acerca da identificação de padrões alimentares e sua associação com fatores socio- econômicos e ambiente alimentar são escassos no Brasil, sobretudo com populações de áreas de baixa renda. Assim, objetivou-se estudar a associação entre padrão alimentar, fatores demográficos e socioeconômicos e o ambiente alimentar em população de uma cidade do Sul do Brasil.

\section{Metodologia}

É um estudo transversal, de base populacional, realizado no território de abrangência de uma unidade básica de saúde (UBS) da área central do município de Porto Alegre (RS). Integra o projeto de pesquisa "Estudo dos determinantes sociais e ambientais da alimentação e nutrição: uma abordagem ecossocial", que foi aprovada pelo Comitê de Ética e Pesquisa da Universidade Federal do Rio Grande do Sul sob número CAAE 46934015.3.0000.5347. A área central de Porto Alegre possui cerca de 260 mil moradores ${ }^{12}$ e é atendida por três unidades básicas de saúde. A população adstrita à UBS estudada é de aproximadamente 12 mil famílias. Parte delas vive em quatro áreas de menor condição socioeconômica (renda per capita média de R\$1.700,00) e bem delimitadas geograficamente (favelas), enquanto o restante das famílias vive em áreas de maior renda (renda per capita média de $\mathrm{R} \$ 4.000,00)^{13}$.

Para garantir esses diferentes estratos socioeconômicos e ambientes alimentares na amostra, realizou-se uma amostragem do tipo proporcional, que incluísse a mesma proporção de indivíduos nas áreas de maior e menor nível socioeconômico. Nas áreas de menor condição socioeconômica, que possuem cerca de 250 famílias, foram incluídos todos os participantes elegíveis que aceitaram participar do estudo. E para manter a proporcionalidade, nas áreas de maior renda, procurou-se incluir o mesmo número de indivíduos. Foram incluídos no estudo moradores com idade entre 20 e 70 anos de ambos os sexos. Não foram incluídos indivíduos com limitações físicas ou mentais, que impossibilitasse a obtenção de dados, e as gestantes. Foi incluído apenas um indivíduo por residência e procurouse alternar o sexo dos participantes a cada residência incluída.

A coleta de dados ocorreu entre outubro de 2018 e junho de 2019. Primeiramente, com a ajuda dos agentes comunitários de saúde (ACS), foram mapeadas as áreas e identificadas as residências nos territórios por meio de mapas e endereços. Depois disso, a equipe de pesquisa se dirigia ao território, identificando os indivíduos que 
atendiam aos critérios de inclusão e realizando o convite para participação na pesquisa. As entrevistas eram feitas nesse momento ou agendadas, e ocorriam, preferencialmente, nos domicílios, ou na UBS, quando os participantes solicitavam.

Utilizou-se um questionário padronizado para obtenção de dados socioeconômicos e demográficos, contendo as seguintes variáveis: sexo (feminino/masculino), idade (anos), raça/cor da pele auto declarada e categorizada conforme o censo do IBGE de $2010^{12}$ (branca/preta/parda/ amarela/indígena), escolaridade (não estudou/ ensino fundamental completo ou incompleto/ ensino médio completo ou incompleto/ensino superior completo/pós-graduação), estado civil (solteiro/união estável/casado/viúvo/separado/ divorciado), renda familiar mensal referida em faixas de salários-mínimos (< $1 \mathrm{SM} / 1$ a $2 \mathrm{SM} / 2$ a $3 \mathrm{SM} / 3$ a $4 \mathrm{SM} / 4$ a $5 \mathrm{SM} />5 \mathrm{SM}$ ), recebimento de benefícios (não recebe/Bolsa Família/aposentadoria ou pensão/Benefício de Prestação Continuada - BPC/outros) e número de moradores no domicílio $(\leq 3 \mathrm{a} \geq 6)$.

Para avaliar o ambiente alimentar, foi utilizado um questionário com os sete itens alimentares mais consumidos pela população brasileira, a saber: produtos industrializados (biscoitos, refrigerantes e macarrão instantâneo), frutas, verduras e legumes, carnes, pães, arroz e feijão) ${ }^{9}$. $\mathrm{O}$ instrumento permitiu investigar o local de compra de cada item (feira/sacolão, supermercado, mercado, mercado público, atacadão, confeitaria/padaria, bar, armazém, açougue, horta em casa, horta comunitária, doações - familiares, vizinhos, instituições - e outros) e a forma de deslocamento utilizada (a pé, de bicicleta, carro/ moto, transporte público). Além disso, o local de realização de refeições foi identificado por meio de um questionário que verificava inicialmente se o indivíduo almoçava e jantava e, em caso de resposta afirmativa, questionava qual era o local em que costumava se alimentar (casa, trabalho, lancheria, restaurante, outro).

O consumo de alimentos foi avaliado por meio de um questionário de frequência alimen$\operatorname{tar}$ (QFA), que a princípio continha 65 alimentos, adaptado para 85 itens, conforme a cultura local. A lista de alimentos do instrumento foi construída a partir de dados de consumo de registros alimentares de adultos de Niterói $(\mathrm{RJ})^{14} \mathrm{e}$ validado para a população da região metropolitana de Porto Alegre (RS $)^{15}$. Os alimentos foram investigados em relação à frequência de consumo (de zero a sete vezes) ao dia, semana, mês ou ano nos últimos 12 meses.
A entrada de dados foi feita no programa EpiData, versão 3.1, em dupla entrada. As análises dos dados foram realizadas nos programas Stata, versão 12.0, e SPSS (Statistical Package for the Social Sciences), versão 18.0.

A análise dos padrões alimentares (PA) foi realizada por metodologia a posteriori, por meio da agregação de dados empíricos de alimentos com base em análise estatística. Esse método se caracteriza por utilizar técnicas multivariadas para identificar similaridades de hábitos alimentares ou de grupos de alimentos consumidos baseadas em suas interrelações ${ }^{16-18}$.

Previamente à identificação dos PA, as diferentes frequências de consumo dos itens alimentares foram transformadas em frequência de consumo anual, inclusive para os alimentos consumidos apenas na época. Depois se verificou a frequência de consumo de cada item alimentar, quando foram excluídos aqueles com consumo abaixo de $5 \%{ }^{19}$. Posteriormente, os itens alimentares foram agregados em 48 grupos, de acordo com suas correlações estatísticas ( $\mathrm{p} \leq 0,05$ ), semelhanças nutricionais e culturais. Outros alimentos foram mantidos sem agregação, seja porque não fazia sentido agrupá-los (adoçante artificial) ou porque poderiam ser indicativos de um determinado padrão alimentar, como arroz e feijão ${ }^{20}$.

A derivação dos PA foi realizada por meio da análise de componentes principais (ACP), considerando-se as exigências teóricas para a sua realização. Assim, para verificar a aplicabilidade do método, foi estimado o coeficiente de Kaiser -Mayer-Olkin (KMO). A medida desse teste indica a força da relação entre as variáveis, sendo que um valor $\geq 0,60$ é considerado adequado. Além disso, foi realizado o teste de esfericidade de Bartlett (Bartlett's test of sphericity, BTS) para testar a hipótese nula, ou seja, de inexistência de relação entre as variáveis. $O$ valor $p<0,05$ indicava adequação para a realização da análise dos dados. No cálculo de poder amostral para identificação dos padrões alimentares foi seguida a regra proposta por Hair e colaboradores ${ }^{21}$, isto é, cinco indivíduos para cada item alimentar incluído na análise de componentes principais.

No intuito de examinar a estrutura fatorial exploratória do QFA, realizou-se a rotação ortogonal Varimax. O número de fatores a extrair foi definido conforme o gráfico da variância (ScreePlot), onde os pontos de maior declive indicaram o número apropriado de componentes a reter, e também por meio do critério de Kaiser, sendo aceitos autovalores maiores que 1 . Os itens ali- 
mentares que apresentaram carga fatorial absoluta $\geq 0,30$ foram considerados de contribuição significativa para determinado fator. Os padrões alimentares foram nomeados de acordo com os alimentos que mais carregaram em cada fator e segundo aspectos culturais. Cada um dos padrões derivados foi dividido em tercis e dicotomizado em tercil 3 (maior consumo) versus tercis 1 e 2 (menor consumo) $)^{22,23}$.

As razões de prevalência brutas e ajustadas e os intervalos de $95 \%$ de confiança (IC 95\%) foram obtidos por meio da regressão de Poisson com variância robusta. Foram incluídas no modelo multivariável aquelas variáveis que apresentaram nível de significância de até $20 \%$ nas análises brutas. A análise multivariada seguiu o modelo conceitual de análise: no primeiro nível foram incluídas as variáveis demográficas, e no segundo nível as socioeconômicas, sendo mantidas apenas as variáveis com p-valor $<0,20$. Após ajuste, as variáveis com $p$-valor $\leq 0,05$ foram consideradas associadas aos padrões alimentares.

\section{Resultados}

O total de participantes do estudo foi de 400 indivíduos. Primeiro foram incluídos todos os moradores das áreas de menor condição socioeconômica que aceitaram participar do estudo (201). Depois foram selecionados 199 moradores das áreas de maior poder aquisitivo.

$\mathrm{Na}$ Tabela 1 estão descritas as características gerais da população e estratificadas por área de moradia. A maior parte dos pesquisados era do sexo feminino e tinha idade média de 47 anos $(\mathrm{DP}=13,98)$. A maioria referiu viver sem companheiro, ter cor da pele branca, escolaridade de ensino médio completo, renda familiar de três a cinco salários-mínimos e morar em domicílios com três ou menos moradores. Metade dos entrevistados recebia algum benefício social. As áreas de menor condição socioeconômica possuem moradores mais jovens, mais frequentemente de cor da pele parda e negra, com menor escolaridade e renda, mais frequentemente beneficiários de programas sociais e com maior número de moradores por domicílio, quando comparadas com as de maior poder aquisitivo.

A Tabela 2 apresenta os quatro padrões alimentares identificados no estudo (saudável, tradicional, carboidratos e açúcares refinados $e$ fast food), com os itens que os compõem, carga fatorial e percentual de variância explicada. Tanto o coeficiente KMO quanto o teste de esfericidade de Bartlett indicaram que as correlações entre os itens eram suficientes e adequadas para se proceder a análise. O PA saudável apresentou o maior percentual de explicação da variância $(10,84 \%)$, ou seja, é o que melhor representa o consumo na população estudada.

Na Tabela 3 estão descritas as prevalências de alto consumo de cada um dos padrões alimentares, segundo as características demográficas e sociais dos indivíduos. O alto consumo do PA saudável foi associado às mulheres, indivíduos que recebiam ou moravam com alguém que recebia aposentadoria, pensão, BPC ou outro benefício social, e que residiam nas áreas de maior condição socioeconômica. O alto consumo do PA tradicional foi associado a indivíduos jovens, pardos, com menor escolaridade, com menor renda, que recebiam ou moravam com alguém que recebia benefício social oriundo do Programa Bolsa Família (PBF) e residiam nas áreas de menor condição socioeconômica em domicílios com seis ou mais moradores. $\mathrm{O}$ alto consumo do PA carboidratos e açúcares refinados foi associado aos mais jovens, de cor da pele preta, com baixa escolaridade, com menor renda, que recebiam ou moravam com alguém que recebia benefício social proveniente do PBF e residiam nas áreas de maior vulnerabilidade, em domicílios com seis ou mais moradores. Por fim, o alto consumo do PA fast food foi associado a ser mais jovem, branco, com maior nível de ensino, ter renda mensal familiar acima de cinco salários-mínimos e residir nas áreas de maior renda.

Após ajustes, o PA saudável esteve associado diretamente ao sexo feminino e à renda mensal familiar acima de cinco salários-mínimos, e inversamente a residir em domicílios com quatro a cinco moradores. O PA tradicional associou-se diretamente a ser preto ou pardo, a residir em domicílios com seis ou mais moradores, e associouse inversamente a residir em áreas de maior condição socioeconômica e a idade e escolaridade. O PA carboidratos e açúcares refinados associouse diretamente à cor da pele parda e preta e ao maior número de moradores no domicílio. Por outro lado, apresentou associação inversa com a idade e as áreas de maior condição socioeconômica. Por fim, o PA fast food esteve diretamente associado à maior escolaridade e inversamente a idade e cor da pele parda ou preta (Tabela 4).

Na Tabela 5 estão descritas as frequências de distribuição das variáveis de ambiente alimentar e suas associações com os padrões alimentares. Indivíduos com maior adesão ao PA tradicional compravam com maior frequência frutas, verdu- 
Tabela 1. Descrição da amostra e variáveis sociodemográficas estratificadas segundo a área de moradia de indivíduos em uma cidade do Sul do Brasil. Porto Alegre, RS, 2018-2019 ( $\mathrm{n}=400)$.

\begin{tabular}{|c|c|c|c|c|}
\hline Variáveis & $\mathbf{n}(\%)$ & $\begin{array}{c}\text { Área de moradia } \\
\text { Menor condição } \\
\text { socioeconômica } \\
\text { n (\%) }\end{array}$ & $\begin{array}{c}\text { Área de moradia } \\
\text { Maior condição } \\
\text { socioeconômica } \\
\text { n (\%) }\end{array}$ & $\begin{array}{c}\text { Valor } \\
\text { de } p\end{array}$ \\
\hline \multicolumn{5}{|l|}{ Sexo } \\
\hline Masculino & $100(25,0)$ & $46(22,9)$ & $54(27,1)$ & ${ }^{\star} 0,32$ \\
\hline Feminino & $300(75,0)$ & $155(77,1)$ & $145(72,9)$ & \\
\hline \multicolumn{5}{|l|}{ Idade (anos) } \\
\hline $20-36$ & $105(26,3)$ & $68(33,8)$ & $37(18,6)$ & \\
\hline $37-49$ & $97(24,3)$ & $56(27,9)$ & $41(20,6)$ & ${ }^{*}<0,001$ \\
\hline $50-59$ & $110(27,5)$ & $53(26,4)$ & $57(28,6)$ & \\
\hline$\geq 60$ & $88(22,0)$ & $24(11,9)$ & $64(32,2)$ & \\
\hline \multicolumn{5}{|l|}{ Cor da pele } \\
\hline Branca & $249(62,3)$ & $95(47,3)$ & $154(77,4)$ & \\
\hline Preta & $78(19,5)$ & $55(27,4)$ & $23(11,6)$ & ${ }^{*}<0,001$ \\
\hline Parda & $73(18,3)$ & $51(25,4)$ & $22(11,1)$ & \\
\hline \multicolumn{5}{|l|}{ Estado civil } \\
\hline Com companheiro & $149(37,3)$ & $77(38,3)$ & $72(36,2)$ & ${ }^{\star} 0,66$ \\
\hline Sem companheiro & $251(62,8)$ & $124(61,7)$ & $127(63,8)$ & \\
\hline \multicolumn{5}{|l|}{ Escolaridade } \\
\hline EF incompleto & $73(18,5)$ & $63(32,1)$ & $10(5,0)$ & \\
\hline EF completo & $73(18,5)$ & $53(27,0)$ & $20(10,1)$ & ${ }^{*}<0,001$ \\
\hline EM completo & $146(37,0)$ & $70(35,7)$ & $76(38,2)$ & \\
\hline ES completo + pós-graduação & $103(26,0)$ & $10(5,1)$ & $93(46,7)$ & \\
\hline \multicolumn{5}{|l|}{ Renda (SM) } \\
\hline$<1$ & $22(5,5)$ & $19(9,5)$ & $3(1,5)$ & \\
\hline 1 a 2 & $112(28,1)$ & $83(41,5)$ & $29(14,6)$ & ${ }^{*}<0,001$ \\
\hline 3 a 5 & $193(48,4)$ & $91(45,5)$ & $102(51,3)$ & \\
\hline$>5$ & $72(18,0)$ & $7(3,5)$ & $65(32,7)$ & \\
\hline \multicolumn{5}{|l|}{ Benefício social } \\
\hline Não recebe & $201(50,3)$ & $99(49,3)$ & $102(51,3)$ & \\
\hline Bolsa Família & $39(9,8)$ & $36(17,9)$ & $3(1,5)$ & ${ }^{*}<0,001$ \\
\hline Aposen./pensão/BPC + Outros & $160(40,0)$ & $66(32,8)$ & $94(47,2)$ & \\
\hline \multicolumn{5}{|l|}{ No de moradores no domicílio } \\
\hline$\leq 3$ & $261(65,3)$ & $116(57,7)$ & $145(72,9)$ & \\
\hline $4-5$ & $102(25,5)$ & $58(28,9)$ & $44(22,1)$ & ${ }^{*}<0,001$ \\
\hline$\geq 6$ & $37(9,3)$ & $27(13,4)$ & $10(5,0)$ & \\
\hline
\end{tabular}

Legenda: $\mathrm{EF}$ = ensino fundamental; $\mathrm{EM}$ = ensino médio; $\mathrm{ES}=$ ensino superior; $\mathrm{SM}$ = salário-mínimo; Aposen.= aposentadoria; $\mathrm{BPC}=$ Benefício de Prestação Continuada.${ }^{*}$ Teste qui-quadrado de Pearson.

Fonte: Autores.

ras e legumes no supermercado, mercado ou atacadão e se deslocavam a pé ou de bicicleta para a compra de produtos industrializados, carnes e pães. Em relação aos indivíduos com maior consumo do PA carboidratos e açúcares refinados, o local de compra mais frequente para pães foi confeitaria/padaria, bar ou armazém. Já aqueles com maior consumo do PA fast food, o deslocamento para a compra da maioria dos itens alimentares ocorreu de carro/moto ou transporte público. Fazer as principais refeições em casa esteve asso- ciado ao PA tradicional, e realizar o almoço ou jantar fora de casa esteve associado aos padrões carboidratos e açúcares refinados e fast food.

\section{Discussão}

Foram identificados quatro padrões alimentares na população, denominados como saudável, tradicional, carboidratos e açúcares refinados e fast food. Ser mulher e possuir maior renda esteve 
Tabela 2. Padrões alimentares com os itens que os compõem, carga fatorial e percentual de variância explicada de indivíduos em uma cidade do Sul do Brasil. Porto Alegre, RS, 2018-2019 ( $\mathrm{n}=400)$.

\begin{tabular}{|c|c|c|c|}
\hline Padrões alimentares & Itens alimentares & $\begin{array}{l}\text { Carga } \\
\text { fatorial }\end{array}$ & $\begin{array}{c}\text { \% variância } \\
\text { explicada }\end{array}$ \\
\hline PA (saudável) & $\begin{array}{l}\text { Alface, agrião } \\
\text { Brócolis, couve-flor, repolho } \\
\text { Moranga, cenoura, beterraba, tomate } \\
\text { Sopa de legumes + chuchu e berinjela } \\
\text { Uva + melancia + manga + mamão + suco natural } \\
\text { Batata doce + polenta mole } \\
\text { Maçã } \\
\text { Laranja } \\
\text { Banana } \\
\text { Arroz integral + pão integral } \\
\text { Aipim/inhame }\end{array}$ & $\begin{array}{l}0,678 \\
0,659 \\
0,610 \\
0,590 \\
0,563 \\
0,523 \\
0,435 \\
0,410 \\
0,356 \\
0,345 \\
0,317\end{array}$ & 10,84 \\
\hline PA (tradicional) & $\begin{array}{l}\text { Arroz branco } \\
\text { Feijão preto } \\
\text { Bife frito }+ \text { frango frito } \\
\text { Carne moída } \\
\text { Carne de gado assada } \\
\text { Farofa }+ \text { polenta frita } \\
\text { Macarrão (massas) } \\
\text { Batata cozida ou assada } \\
\text { Carne seca/charque + carne de porco + almôndega + } \\
\text { fígado de boi } \\
\text { Purê de batata }\end{array}$ & $\begin{array}{l}0,586 \\
0,463 \\
0,452 \\
0,436 \\
0,429 \\
0,419 \\
0,408 \\
0,396 \\
0,378 \\
0,323\end{array}$ & 7,35 \\
\hline $\begin{array}{l}\text { PA (carboidratos e } \\
\text { açúcares refinados) }\end{array}$ & $\begin{array}{l}\text { Bolos (simples e com cobertura) + biscoitos (doce, } \\
\text { salgado e recheado) } \\
\text { Pão francês + pão de forma } \\
\text { Suco em pó + suco caixa } \\
\text { Açúcar + achocolatado + bala/chiclete com açúcar } \\
\text { Refrigerante normal + refrigerante zero } \\
\text { Maionese + margarina } \\
\text { Rapadura + chimia + doce de leite } \\
\text { Sorvete } \\
\text { Iogurte + leites (integral, semidesnatado, desnatado) }\end{array}$ & $\begin{array}{l}0,574 \\
0,565 \\
0,540 \\
0,501 \\
0,499 \\
0,471 \\
0,363 \\
0,357 \\
0,314\end{array}$ & 4,86 \\
\hline PA (fast food) & $\begin{array}{l}\text { Pizza } \\
\text { Xis + salgado frito + macarrão instantâneo + salsicha } \\
\text { Chocolate/bombom } \\
\text { Batata frita ou palha } \\
\text { Salgado assado (empada, pão de queijo) } \\
\text { Cerveja/vinho + destilados (cachaça, uísque) } \\
\text { Outras leguminosas (ervilha, soja, grão de bico) }\end{array}$ & $\begin{array}{l}0,672 \\
0,557 \\
0,544 \\
0,541 \\
0,510 \\
0,386 \\
0,362\end{array}$ & 4,29 \\
\hline $\begin{array}{l}\text { Total de variância } \\
\text { explicada }\end{array}$ & & & 27,34 \\
\hline
\end{tabular}

KMO: 0,731; teste de esfericidade de Bartlett: $<0,001$.

Fonte: Autores.

associado ao consumo de um padrão alimentar mais saudável. Possuir menos idade, cor da pele parda ou negra, menor escolaridade, maior número de moradores na residência e morar em áreas de menor renda estiveram associados ao consumo dos padrões considerados menos sau- dáveis ou com menor participação de frutas e verduras. Além disso, comprar os alimentos em grandes supermercados deslocando-se a pé ou de bicicleta e fazer as principais refeições em casa esteve associado ao padrão alimentar tradicional brasileiro. Por outro lado, deslocar-se para as 
Tabela 3. Prevalência de alto consumo aos padrões alimentares de indivíduos em uma cidade do Sul do Brasil. Porto Alegre, RS, 2018-2019 $(\mathrm{n}=400)$.

\begin{tabular}{|c|c|c|c|c|c|c|c|c|}
\hline Variáveis (n) & $\begin{array}{c}\text { PA } \\
\text { saudável } \\
\text { n (\%) }\end{array}$ & $\begin{array}{l}\text { Valor } \\
\text { de p }\end{array}$ & $\begin{array}{c}\text { PA trad. } \\
\text { n }(\%)\end{array}$ & $\begin{array}{l}\text { Valor } \\
\text { de p }\end{array}$ & $\begin{array}{c}\text { PA carb./ } \\
\text { acúc. } \\
\text { n (\%) }\end{array}$ & $\begin{array}{l}\text { Valor } \\
\text { de p }\end{array}$ & $\begin{array}{c}\text { PA fast } \\
\text { food } \\
\text { n (\%) }\end{array}$ & $\begin{array}{l}\text { Valor } \\
\text { de p }\end{array}$ \\
\hline \multicolumn{9}{|l|}{ Sexo } \\
\hline Masculino (100) & $20(20,0)$ & ${ }^{*}<0,001$ & $37(37,0)$ & ${ }^{\star} 0,39$ & $40(40,0)$ & ${ }^{*} 0,09$ & $39(39,0)$ & ${ }^{*} 0,17$ \\
\hline Feminino (300) & $114(38,0)$ & & $97(32,3)$ & & $93(31,0)$ & & $95(31,7)$ & \\
\hline \multicolumn{9}{|l|}{ Idade (anos) } \\
\hline $20-36(105)$ & $32(30,5)$ & & $43(41,0)$ & & $42(40,0)$ & & $42(40,0)$ & \\
\hline $37-49(97)$ & $28(28,9)$ & & $38(39,2)$ & & $38(39,2)$ & & $38(39,2)$ & \\
\hline $50-59(110)$ & $39(35,5)$ & ${ }^{* *} 0,37$ & $32(29,1)$ & ${ }^{* *} 0,03$ & $32(29,1)$ & ${ }^{* *} 0,04$ & $34(30,9)$ & ${ }^{* *} 0,04$ \\
\hline$\geq 60(88)$ & $35(39,8)$ & & $21(23,9)$ & & $21(23,9)$ & & $20(22,7)$ & \\
\hline \multicolumn{9}{|l|}{ Cor da pele } \\
\hline Branca (249) & $90(36,1)$ & & $64(25,7)$ & & $65(26,1)$ & & $98(39,4)$ & \\
\hline Preta $(78)$ & $25(32,1)$ & ${ }^{*} 0,26$ & $36(46,2)$ & ${ }^{*}<0,001$ & $38(48,7)$ & ${ }^{*}<0,001$ & $16(20,5)$ & ${ }^{*}<0,001$ \\
\hline Parda (73) & $19(26,0)$ & & $34(46,6)$ & & $30(41,1)$ & & $20(27,4)$ & \\
\hline \multicolumn{9}{|l|}{ Estado civil } \\
\hline C/ comp. (149) & $48(32,2)$ & ${ }^{\star} 0,67$ & $46(30,9)$ & ${ }^{\star} 0,39$ & $46(30,9)$ & ${ }^{\star} 0,43$ & $47(31,5)$ & ${ }^{*} 0,52$ \\
\hline S/ comp. (251) & $86(34,3)$ & & $88(35,1)$ & & $87(34,7)$ & & $87(34,7)$ & \\
\hline \multicolumn{9}{|l|}{ Escolaridade } \\
\hline EF incomp. (73) & $24(32,9)$ & & $46(63,0)$ & & $33(45,2)$ & & $13(17,8)$ & \\
\hline EF comp. (73) & $20(27,4)$ & & $30(41,1)$ & & $30(41,1)$ & & $20(27,4)$ & \\
\hline EM comp. (146) & $46(31,5)$ & ${ }^{* *} 0,20$ & $46(31,5)$ & ${ }^{*}<0,001$ & $54(37,0)$ & ${ }^{* *}<0,001$ & $48(32,9)$ & ${ }^{*}<0,001$ \\
\hline $\mathrm{ES}+\mathrm{PG}(103)$ & $43(41,7)$ & & $10(9,7)$ & & $13(12,6)$ & & $51(49,5)$ & \\
\hline \multicolumn{9}{|l|}{ Renda (SM) } \\
\hline$<1(22)$ & $4(18,2)$ & & $13(59,1)$ & & $11(50,0)$ & & $06(27,3)$ & \\
\hline 1 a $2(112)$ & $32(28,6)$ & & $53(47,3)$ & & $43(38,4)$ & & $26(23,2)$ & \\
\hline 3 a 5 (193) & $66(34,2)$ & ${ }^{* *} 0,08$ & $54(28,0)$ & ${ }^{* *}<0,001$ & $65(33,7)$ & ${ }^{* *}<0,001$ & $65(33,7)$ & $* *<0,001$ \\
\hline$>5(72)$ & $31(43,1)$ & & $14(19,4)$ & & $13(18,1)$ & & $37(51,4)$ & \\
\hline \multicolumn{9}{|l|}{ Benef. social } \\
\hline Não recebe (201) & $70(34,8)$ & & $59(29,4)$ & & $60(29,9)$ & & $76(37,8)$ & \\
\hline Bolsa Fam. (39) & $6(15,4)$ & ${ }^{\star} 0,04$ & $27(69,2)$ & ${ }^{*}<0,001$ & $25(64,1)$ & ${ }^{*}<0,001$ & $12(30,8)$ & ${ }^{\star} 0,18$ \\
\hline Ap. outros (160) & $58(36,3)$ & & $48(30,0)$ & & $48(30,0)$ & & $46(28,8)$ & \\
\hline \multicolumn{9}{|l|}{ Área moradia } \\
\hline Menor CSE (201) & $56(27,9)$ & ${ }^{\star} 0,01$ & $106(52,7)$ & ${ }^{*}<0,001$ & $101(50,2)$ & ${ }^{*}<0,001$ & $56(27,9)$ & ${ }^{\star} 0,01$ \\
\hline Maior CSE (199) & $78(39,2)$ & & $28(14,1)$ & & $32(16,1)$ & & $78(39,2)$ & \\
\hline \multicolumn{9}{|c|}{ No moradores por domicílio } \\
\hline$\leq 3(261)$ & $96(36,8)$ & & $68(26,1)$ & & $71(27,2)$ & & $85(32,6)$ & \\
\hline $4-5(102)$ & $29(28,4)$ & ${ }^{* *} 0,14$ & $40(39,2)$ & ${ }^{* *}<0,001$ & $39(38,2)$ & ${ }^{* *}<0,001$ & $39(38,2)$ & ${ }^{* *} 0,40$ \\
\hline$\geq 6(37)$ & $9(24,3)$ & & $26(70,3)$ & & $23(62,2)$ & & $10(27,0)$ & \\
\hline
\end{tabular}

Legenda: $\mathrm{EF}$ = ensino fundamental; $\mathrm{EM}=$ ensino médio; $\mathrm{ES}=$ ensino superior; $\mathrm{SM}$ = salários-mínimos; $\mathrm{CSE}$ = condição socioeconômica; $\mathrm{BPC}=$ Benefício de Prestação Continuada. ${ }^{*}$ Teste qui-quadrado de Pearson. ${ }^{*}$ Teste de linearidade.

Fonte: Autores.

compras de carro ou ônibus e realizar as principais refeições fora de casa esteve associado a um padrão de risco com alimentos ultraprocessados.

Em relação à composição, o PA saudável era rico em frutas, hortaliças e cereais integrais, já o PA tradicional era composto por alimentos consumidos cotidianamente pelos brasileiros, como arroz, feijão, massa, batata e carne vermelha. Ao mesmo tempo, foram identificados dois padrões alimentares predominantemente compostos por alimentos ultraprocessados. Esses resultados corroboram dados de outras pesquisas no Brasil que demonstraram um aumento no consumo de alimentos ricos em carboidratos, açúcares e gor- 
Tabela 4. Razões de prevalência (RP) brutas e ajustadas e intervalos de $95 \%$ de confiança (IC 95\%) para alto consumo ao padrão alimentar de acordo com variáveis socioeconômicas e demográficas de indivíduos em uma cidade do Sul do Brasil. Porto Alegre, RS, 2018-2019 $(\mathrm{n}=400)$.

\begin{tabular}{|c|c|c|c|c|}
\hline \multirow{2}{*}{ Variáveis } & PA saudável & PA tradicional & $\begin{array}{c}\text { PA carb.e } \\
\text { açúcares refin. }\end{array}$ & PA fast food \\
\hline & $\begin{array}{l}\text { RP Ajustada } \\
\text { IC } 95 \%\end{array}$ & $\begin{array}{l}\text { RP Ajustada } \\
\text { IC 95\% }\end{array}$ & $\begin{array}{l}\text { RP Ajustada } \\
\text { IC } 95 \%\end{array}$ & $\begin{array}{l}\text { RP Ajustada } \\
\text { IC } 95 \%\end{array}$ \\
\hline Sexo & ${ }^{*} \mathrm{p}=<0,001$ & & ${ }^{\star} \mathrm{p}=0,11$ & ${ }^{*} \mathrm{p}=0,22$ \\
\hline Masculino & 1,00 & - & 1,00 & 1,00 \\
\hline Feminino & $1,15(1,06-1,24)$ & & $0,94(0,87-1,01)$ & $0,94(0,87-1,02)$ \\
\hline Idade (anos) & ${ }^{* *} \mathrm{p}=0,09$ & ${ }^{*} \mathrm{p}=<0,001$ & ${ }^{* *} \mathrm{p}=<0,001$ & ${ }^{* *} \mathrm{p}=<0,001$ \\
\hline $20-36$ & 1,00 & 1,00 & 1,00 & 1,00 \\
\hline $37-49$ & $0,99(0,89-1,09)$ & $0,99(0,90-1,09)$ & $1,00(0,91-1,09)$ & $0,98(0,89-1,08)$ \\
\hline $50-59$ & $1,04(0,94-1,14)$ & $0,93(0,85-1,03)$ & $0,94(0,86-1,03)$ & $0,90(0,82-0,99)$ \\
\hline$\geq 60$ & $1,07(0,97-1,18)$ & $0,88(0,80-0,97)$ & $0,88(0,80-0,97)$ & $0,86(0,78-0,95)$ \\
\hline Cor da pele & & ${ }^{\star} \mathrm{p}=<0,001$ & ${ }^{\star} \mathrm{p}=<0,001$ & ${ }^{\star} \mathrm{p}=<0,001$ \\
\hline Branca & - & 1,00 & 1,00 & 1,00 \\
\hline Preta & & $1,15(1,05-1,26)$ & $1,17(1,07-1,27)$ & $0,85(0,78-0,93)$ \\
\hline Parda & & $1,15(1,06-1,26)$ & $1,10(1,01-1,21)$ & $0,90(0,82-0,98)$ \\
\hline \multicolumn{5}{|l|}{ Estado civil } \\
\hline Com companheiro & - & - & - & - \\
\hline \multicolumn{5}{|l|}{ Sem companheiro } \\
\hline Escolaridade & ${ }^{* *} \mathrm{p}=0,53$ & ${ }^{* *} \mathrm{p}=<0,001$ & ${ }^{* *} \mathrm{p}=0,74$ & ${ }^{* *} \mathrm{p}=0,04$ \\
\hline EF incompleto & 1,00 & 1,00 & 1,00 & 1,00 \\
\hline EF completo & $0,93(0,83-1,04)$ & $0,89(0,80-0,98)$ & $0,98(0,88-1,09)$ & $1,06(0,95-1,18)$ \\
\hline EM completo & $0,94(0,84-1,04)$ & $0,87(0,79-0,96)$ & $1,01(0,91-1,11)$ & $1,07(0,96-1,18)$ \\
\hline $\mathrm{ES}+\mathrm{PG}$ & $0,95(0,83-1,09)$ & $0,81(0,71-0,92)$ & $0,94(0,83-1,06)$ & $1,14(1,00-1,29)$ \\
\hline Renda & ${ }^{* *} \mathrm{p}=0,04$ & ${ }^{* *} \mathrm{p}=0,61$ & ${ }^{* *} \mathrm{p}=0,71$ & ${ }^{* *} \mathrm{p}=0,13$ \\
\hline$<1 \mathrm{SM}$ & 1,00 & 1,00 & 1,00 & 1,00 \\
\hline 1 a 2 SM & $1,08(0,91-1,28)$ & $1,00(0,87-1,14)$ & $0,99(0,85-1,17)$ & $0,91(0,77-1,09)$ \\
\hline 3 a $5 \mathrm{SM}$ & $1,14(0,96-1,35)$ & $0,95(0,83-1,09)$ & $1,03(0,88-1,20)$ & $0,96(0,81-1,15)$ \\
\hline$>5 \mathrm{SM}$ & $1,19(0,98-1,44)$ & $0,99(0,85-1,15)$ & $1,01(0,85-1,21)$ & $1,03(0,85-1,26)$ \\
\hline Benefício social & & & & ${ }^{*} \mathrm{p}=0,51$ \\
\hline Não recebe & - & - & - & 1,00 \\
\hline Bolsa Família & & & & $1,03(0,91-1,17)$ \\
\hline Apos. + outros & & & & $0,96(0,89-1,04)$ \\
\hline Área moradia & ${ }^{*} \mathrm{p}=0,20$ & ${ }^{*} \mathrm{p}=<0,001$ & ${ }^{*} \mathrm{p}=<0,001$ & ${ }^{*} \mathrm{p}=0,72$ \\
\hline Menor CSE & 1,00 & 1,00 & 1,00 & 1,00 \\
\hline Maior CSE & $1,05(0,96-1,15)$ & $0,83(0,75-0,91)$ & $0,82(0,75-0,90)$ & $1,01(0,93-1,10)$ \\
\hline $\mathrm{N}^{\circ}$ de moradores & ${ }^{\star *} \mathrm{p}=0,04$ & ${ }^{* *} \mathrm{p}=<0,001$ & ${ }^{* *} \mathrm{p}=<0,001$ & \\
\hline$\leq 3$ & 1,00 & 1,00 & 1,00 & - \\
\hline $4-5$ & $0,92(0,85-1,00)$ & $1,06(0,98-1,14)$ & $1,04(0,96-1,12)$ & \\
\hline$\geq 6$ & $0,93(0,82-1,04)$ & $1,22(1,11-1,34)$ & $1,17(1,05-1,30)$ & \\
\hline
\end{tabular}

Legenda: PA = padrão alimentar; $\mathrm{RP}$ = razão de prevalência; IC 95\% = intervalo de confiança de 95\%; $\mathrm{EF}$ = ensino fundamental; $\mathrm{EM}=$ ensino médio; $\mathrm{ES}=$ ensino superior; $\mathrm{PG}=$ pós-graduação; $\mathrm{SM}$ = salário-mínimo; $\mathrm{BPC}=$ Benefício de Prestação Continuada; CSE $=$ condição socioeconômica. ${ }^{*}$ Teste de Wald. ${ }^{* *}$ Teste de linearidade.

Fonte: Autores.

duras, mas que também encontraram um padrão alimentar baseado em alimentos tradicionalmente consumidos em nosso país, como arroz e feijão.
Quando se considera a composição dos padrões encontrados, embora eventualmente com diferentes denominações, foram similares aos identificados em outras pesquisas realizadas no 
Tabela 5. Locais e formas de deslocamento para compra de alimentos, local de almoço e jantar e associações com os padrões alimentares de indivíduos em uma cidade do Sul do Brasil. Porto Alegre, RS, 2018-2019 ( $\mathrm{n}=400$ ).

\begin{tabular}{|c|c|c|c|c|c|c|c|c|}
\hline Variáveis (n) & $\begin{array}{c}\text { PA } \\
\text { saudável } \\
\text { n (\%) } \\
\end{array}$ & $\begin{array}{c}\text { Valor } \\
\text { de p }\end{array}$ & $\begin{array}{c}\text { PA trad. } \\
\text { n }(\%)\end{array}$ & $\begin{array}{l}\text { Valor } \\
\text { de p }\end{array}$ & $\begin{array}{c}\text { PA carb. e } \\
\text { açúcar. ref. } \\
\text { n (\%) }\end{array}$ & $\begin{array}{c}\text { Valor } \\
\text { de p }\end{array}$ & $\begin{array}{c}\text { PA fast } \\
\text { food } \\
\text { n }(\%)\end{array}$ & $\begin{array}{l}\text { Valor } \\
\text { de p }\end{array}$ \\
\hline \multicolumn{9}{|l|}{ Local da Compra } \\
\hline \multicolumn{9}{|l|}{ Produtos industrializados } \\
\hline Superm.+ mercado + atacadão (390) & $131(33,6)$ & & $132(33,8)$ & & $130(33,3)$ & & $132(33,8)$ & \\
\hline Feira/sacolão + Mercado público (1) & $0(0,0)$ & 0,77 & $0(0,0)$ & 0,59 & $1(100,0)$ & 0,28 & $0(0,00)$ & 0,59 \\
\hline Outros $^{1}(9)$ & $3(33,3)$ & & $2(22,2)$ & & $2(22,2)$ & & $2(22,2)$ & \\
\hline \multicolumn{9}{|l|}{ Frutas } \\
\hline Superm.+ mercado + atacadão (306) & $99(32,4)$ & & $113(36,9)$ & & $109(35,6)$ & & $98(32,0)$ & \\
\hline Feira/sacolão + Mercado público (71) & $28(39,4)$ & 0,49 & $18(25,4)$ & 0,01 & $20(28,2)$ & 0,12 & $26(36,6)$ & 0,44 \\
\hline $\operatorname{Outros}^{1}(23)$ & $7(30,4)$ & & $3(13,0)$ & & $4(17,4)$ & & $10(43,5)$ & \\
\hline \multicolumn{9}{|l|}{ Verduras e legumes } \\
\hline Superm.+ mercado + atacadão (304) & $100(32,9)$ & & $113(37,2)$ & & $108(35,5)$ & & $97(31,9)$ & \\
\hline Feira/sacolão + Mercado público (75) & $29(38,7)$ & 0,50 & $18(24,0)$ & $<0,001$ & $22(29,3)$ & 0,14 & $30(40,0)$ & 0,39 \\
\hline Outros $^{1}(19)$ & $5(26,3)$ & & $2(10,5)$ & & $3(15,8)$ & & $7(36,8)$ & \\
\hline \multicolumn{9}{|l|}{ Carnes } \\
\hline Superm.+ mercado + atacadão (284) & $98(34,5)$ & & $105(37,0)$ & & $93(32,7)$ & & $99(34,9)$ & \\
\hline Feira/sacolão + Mercado público + açougue (97) & $27(27,8)$ & 0,47 & $26(26,8)$ & 0,11 & $35(36,1)$ & 0,77 & $26(26,8)$ & 0,14 \\
\hline $\operatorname{Outros}^{2}(14)$ & $5(35,7)$ & & $3(21,4)$ & & $4(28,6)$ & & $7(50,0)$ & \\
\hline \multicolumn{9}{|l|}{ Pães } \\
\hline Superm.+ mercado + atacadão (276) & $96(34,8)$ & & $84(30,4)$ & & $81(29,3)$ & & $100(36,2)$ & \\
\hline Confeitaria/padaria + Bar + armazém (112) & $32(28,6)$ & 0,23 & $47(42,0)$ & 0,07 & $47(42,0)$ & 0,04 & $32(28,6)$ & 0,15 \\
\hline $\operatorname{Outros}^{3}(12)$ & $6(50,0)$ & & $3(25,0)$ & & $5(41,7)$ & & $2(16,7)$ & \\
\hline \multicolumn{9}{|l|}{ Arroz e feijão } \\
\hline Superm.+ Mercado + Atacadão (378) & $127(33,6)$ & & $131(34,7)$ & & $129(34,1)$ & & $127(33,6)$ & \\
\hline Feira/sacolão + Mercado Público (9) & $5(55,6)$ & 0,06 & $1(11,1)$ & 0,15 & $1(11,1)$ & 0,28 & $2(22,2)$ & 0,64 \\
\hline \multicolumn{9}{|l|}{ Deslocamento } \\
\hline \multicolumn{9}{|l|}{ Produtos industrializados } \\
\hline A pé + bicicleta (235) & $79(33,6)$ & 0,98 & $88(37,4)$ & 0,05 & $80(34,0)$ & 0,62 & $66(28,1)$ & $<0,001$ \\
\hline Carro/moto + transp. público $(164)$ & $55(33,5)$ & & $46(28,0)$ & & $52(31,7)$ & & $68(41,5)$ & \\
\hline \multicolumn{9}{|l|}{ Frutas } \\
\hline A pé + bicicleta $(232)$ & $78(33,6)$ & 0,98 & $84(36,2)$ & 0,20 & $79(34,1)$ & 0,65 & $67(28,9)$ & 0,03 \\
\hline Carro/moto + transp. público (163) & $55(33,7)$ & & $49(30,1)$ & & $52(31,9)$ & & $64(39,3)$ & \\
\hline \multicolumn{9}{|l|}{ Carnes } \\
\hline A pé + bicicleta (189) & $61(32,3)$ & 0,82 & $76(40,2)$ & 0,01 & $61(32,3)$ & 0,59 & $63(33,3)$ & 1,00 \\
\hline Carro/moto + transp. público (204) & $68(33,3)$ & & $58(28,4)$ & & $71(34,8)$ & & $68(33,3)$ & \\
\hline \multicolumn{9}{|l|}{ Pães } \\
\hline A pé + bicicleta (278) & $85(30,6)$ & 0,17 & $107(38,5)$ & $<0,001$ & $97(34,9)$ & 0,33 & $81(29,1)$ & $<0,001$ \\
\hline Carro/moto + transp. público $(117)$ & $44(37,6)$ & & $26(22,2)$ & & $35(29,9)$ & & $52(44,4)$ & \\
\hline $\operatorname{Outros}^{1}(12)$ & $1(8,3)$ & & $2(16,7)$ & & $3(25,0)$ & & $5(41,7)$ & \\
\hline \multicolumn{9}{|l|}{ Arroz e feijão } \\
\hline A pé + bicicleta $(226)$ & $76(33,6)$ & 0,98 & $84(37,2)$ & 0,11 & $78(34,5)$ & 0,59 & $62(27,4)$ & $<0,001$ \\
\hline Carro/moto + transp. público (166) & $56(33,7)$ & & $49(29,5)$ & & $53(31,9)$ & & $69(41,6)$ & \\
\hline \multicolumn{9}{|l|}{ Local da Refeição } \\
\hline \multicolumn{9}{|l|}{ Almoço } \\
\hline Em casa (273) & $92(33,7)$ & 0,90 & $103(37,7)$ & $<0,001$ & $96(35,2)$ & 0,23 & $76(27,8)$ & $<0,001$ \\
\hline Fora de casa ou não faz a refeição (127) & $42(33,1)$ & & $31(24,4)$ & & $37(29,1)$ & & $58(45,7)$ & \\
\hline \multicolumn{9}{|l|}{ Jantar } \\
\hline Em casa $(327)$ & $110(33,6)$ & 0,90 & $108(33,0)$ & 0,67 & $101(30,9)$ & 0,03 & $112(34,3)$ & 0,50 \\
\hline Fora de casa ou não faz a refeição (73) & $24(32,9)$ & & $26(35,6)$ & & $32(43,8)$ & & $22(30,1)$ & \\
\hline
\end{tabular}

Fonte: Autores. 
país, mais especificamente nos estados do Rio Grande do Sul ${ }^{24,25}$, São Paulo ${ }^{26-28}$, Rio de Janeiro ${ }^{29}$, Espírito Santo ${ }^{30}$ e Ceará ${ }^{31}$. Além disso, dados do estudo ELSA-Brasil ${ }^{32}$ e do Inquérito Nacional de Alimentação (INA) 33 também foram incluídos.

Os resultados do presente estudo mostraram que o sexo feminino e maior renda apresentaram associação direta com PA saudável, e esses achados corroboram outras investigações ${ }^{26,28,34-37}$. As mulheres são mais conscientes e valorizam a relação entre alimentação e saúde, além de assumirem as principais responsabilidades pelo ato de $\operatorname{cozinhar}^{38}$. Dados da pesquisa VIGITEL mostraram que o consumo regular de frutas e hortaliças é mais alto entre mulheres $(39,2 \%)$ do que entre homens $(27,7 \%)^{39}$. Além disso, autores reforçam que um nível socioeconômico maior proporciona condições de adquirir os itens alimentares ${ }^{40}$.

Com relação à idade, indivíduos mais velhos apresentaram associação inversa aos padrões alimentares tradicional, carboidratos e açúcares refinados e fast food. Autores como Avelar e Rezende $^{41}$ relacionam o aumento da idade com a preocupação sobre a qualidade da dieta. Estudos ressaltam que o consumo energético proveniente de alimentos ultraprocessados é alto entre adultos e adolescentes, pois consomem maior quantidade de embalados como doces, refrigerantes e biscoitos, produtos ricos em açúcares, gorduras e sódio $^{42-44}$. Dados de duas pesquisas brasileiras recentes mostram que percentuais elevados de calorias consumidas por indivíduos mais jovens foram provenientes dos ultraprocessados, $49,2 \%{ }^{43}$ e $50,6 \%{ }^{44}$. Resultados semelhantes foram encontrados no Canadá $(51,2 \%)^{45}$, nos Estados Unidos $(57,9 \%)^{46}$ e no Reino Unido $(50,4 \%)^{47}$, onde as dietas também continham predominantemente alimentos industrializados.

São encontrados poucos estudos que investigaram a influência da raça/cor da pele no consumo de alimentos na literatura. Estudo que comparou os padrões alimentares de brancos e negros americanos mostrou que o padrão alimentar baseado em carne processada, frituras, grãos refinados, açúcar, margarina, doces e gorduras foi mais frequente entre os negros ${ }^{48}$. No Brasil, análises de pesquisas nacionais mostram que a cor da pele/raça preta ou parda esteve associada ao maior consumo de feijão, carnes e leite com alto teor de gordura e ao menor consumo de frutas e verduras, indicando que a escolha alimentar pode estar associada ao seu valor monetário. Em uma sociedade marcada pelo racismo, a raça/cor da pele, como categoria posição socioeconômica, determina a trajetória de vida dos indivíduos ${ }^{49}$.
Os participantes com maior escolaridade apresentaram associação direta ao PA fast food, assim como em outras pesquisas ${ }^{22,24}$, e inversa ao PA tradicional. Lenz e colaboradores, em estudo realizado com mulheres da região Sul do Brasil, encontraram que o padrão de consumo denominado como de alto risco para DCNT, baseado em doces, biscoitos, queijos, maionese e frituras, entre outros, foi mais frequente entre mulheres com maior escolaridade ${ }^{22}$. Assim, entende-se que maior escolaridade não é sinônimo de escolhas saudáveis. Em revisão sistemática recente, os autores reforçaram a complexidade da relação entre escolaridade e padrão alimentar dos brasileiros, uma vez que a maior escolaridade esteve associada diretamente ao padrão alimentar "duplo", pois os indivíduos se alimentavam de produtos saudáveis mas também consumiam bebidas e alimentos ricos em açúcar e/ou gordura ${ }^{49}$.

A renda esteve associada apenas ao maior consumo do PA saudável, e ao mesmo tempo foi a única variável socioeconômica associada a este PA após ajustes. Os alimentos que compõem o PA, frutas, legumes, verduras e cereais integrais, são habitualmente mais caros do que alimentos ultraprocessados e seu consumo ainda é maior entre os indivíduos de maior renda ${ }^{49}$. A literatura nos mostra que os indivíduos com menor nível socioeconômico geralmente residem em áreas desprovidas de serviços e por isso têm acesso dificultado a locais de compras, como mercados, feiras e sacolões ${ }^{50,51}$.

Além disso, essas áreas mais vulneráveis apresentam menor disponibilidade e acesso a alimentos saudáveis, e quando disponíveis são de baixa qualidade e mais caros. Por outro lado, a exposição dessas pessoas a alimentos não saudáveis é maior, pois a comercialização ocorre em pequenos estabelecimentos e lojas de conveniência ${ }^{50,51}$. Esses achados corroboram o presente estudo, em que os indivíduos que residiam em áreas de menor renda adotavam com maior frequência o PA tradicional ou aquele composto por alimentos ultraprocessados ricos em carboidratos e açúcares refinados.

De acordo com Boyle, Stone-Francisco e Samuels $^{52}$ e Story e colaboradores ${ }^{53}$, o ambiente alimentar pode ser caracterizado como: presença física de alimentos que afetam a dieta dos indivíduos; proximidade de um indivíduo a estabelecimentos de venda de alimentos; distribuição dos comércios de alimentos, serviços de alimentação e qualquer local físico onde possam ser obtidos alimentos; e um sistema conectado que permite o acesso aos alimentos. No presente estudo, 
as pessoas com alto consumo do PA tradicional compravam frutas e verduras em supermercados, mercados e/ou atacados. Nesses locais, esse tipo de alimento normalmente é mais caro do que em feiras e sacolões, fazendo com que priorizem os itens principais da alimentação, diminuindo a compra de frutas e verduras frescas ${ }^{50,51}$. Estudos norte-americanos mostram que produtos industrializados, como lanches, sobremesas e refrigerantes, em geral são adquiridos em grandes redes de supermercados ${ }^{54,55}$. No Brasil, pesquisa indica que $54 \%$ dos gastos familiares com alimentação foram realizados em supermercados ou hipermercados, e no Rio Grande do Sul esse percentual foi de $67 \% 0^{10,13}$.

Quanto às formas de deslocamento para a compra de alimentos, percebe-se que características socioeconômicas podem estar relacionadas às escolhas dos meios de transporte, pois indivíduos com alto consumo do PA tradicional se deslocavam a pé ou de bicicleta, e aqueles com alto consumo do PA fast food utilizavam carro/moto ou transporte público.

Conhecer o local de realização de refeições tem sido pauta de pesquisas que buscam esclarecer melhor a relação entre ambiente alimentar e saúde. Os resultados do estudo mostraram que a maioria dos indivíduos fazia as principais refeições em casa, e almoçar ou jantar fora de casa esteve associado aos padrões carboidratos e açúcares refinados e fast food. Outras pesquisas demonstraram que comer fora de casa está associado ao aumento na participação de alimentos ultraprocessados na dieta de brasileiros, pois refrigerantes e lanches do tipo fast food são algumas das opções mais escolhidas ${ }^{56-58}$.

Dados de estudos nacionais demonstram que a despesa mensal com alimentação fora de casa vem crescendo ao longo dos anos, sendo que na área urbana o percentual de gastos mensais com consumo alimentar fora do domicílio passou de $25,7 \%$ para $33,9 \%$, nas investigações realizadas em 2002-2003 e 2017-2018, respectivamente ${ }^{59}$. Essas mudanças seguem a tendência mundial. O aumento do consumo de alimentos fora de casa pode ser um indicativo de que mudanças na estrutura econômica e social do país estão aumentando as restrições de tempo e, por conseguinte, elevando a demanda por alimentos prontos para consumo $^{56,60}$. A Organização Mundial da Saúde (OMS) ressalta que esse tipo de alimentação possivelmente está associado à maior incidência de DCNT, como diabetes e hipertensão ${ }^{61}$.

O presente estudo possui limitações, entre elas a utilização de um método retrospectivo, podendo levar a erro recordatório. Ressalta-se que a metodologia de avaliação do padrão alimentar possui limitações quanto à subjetividade das decisões que foram tomadas pelos pesquisadores. Por ser um estudo transversal, não se pode assegurar a presença de temporalidade entre exposições e desfechos. Pelo fato de ter sido conduzido em uma população de um território de saúde, a amostra não é representativa da população geral, o que não impede que as associações possam ser generalizadas para outros grupos, desde que haja mecanismos alertando que as mesmas não são válidas.

Por outro lado, este estudo é um dos primeiros a investigar os padrões alimentares de adultos e idosos em áreas centrais de grandes cidades incluindo indivíduos que moram em áreas de baixo nível socioeconômico.

\section{Conclusão}

Variáveis que indicam menor posição socioeconômica estiveram associadas ao maior consumo de PA tradicional e/ou rico em alimentos ultraprocessados, ou seja, padrões alimentares menos saudáveis, porém mais acessíveis em termos monetários.

Por outro lado, a melhor posição socioeconômica esteve associada ao maior consumo de PA saudável, rico em frutas e verduras e/ou um PA fast food, rico em lanches gordurosos, alimentos mais caros. Conclui-se assim que indivíduos que ocupam melhores posições socioeconômicas na sociedade têm oportunidade de escolha entre padrões alimentares saudáveis ou não saudáveis, já os indivíduos de menor posição socioeconômica ficam restritos a padrões alimentares mais baratos, monótonos e quase sempre de baixa qualidade.

O ambiente alimentar interfere na compra de determinados alimentos para o consumo, e assim no padrão alimentar. Os indivíduos que mais aderiram ao padrão alimentar tradicional também realizavam as compras em supermercados, mercados e atacadões, e dessa forma compravam menos frutas, verduras e legumes, provavelmente por serem mais caros, dando lugar à aquisição de alimentos mais básicos, como o arroz e feijão. Realizar o almoço ou jantar fora de casa se associou ao consumo de padrões alimentares menos saudáveis, como o PA fast food e o PA carboidratos.

Os resultados deste estudo poderão contribuir na adequação de políticas de saúde e alimentação e reorganizar as estruturas de logísticas de abastecimento nos territórios, a fim de ajudar a superar diferenças injustas. 


\section{Colaboradores}

CML Cunha, R Canuto, PBZ Rosa participaram do delineamento do estudo, da coleta de dados, análise e interpretação dos dados e redação do artigo. I Schuch participou do delineamento do estudo, da organização da coleta de dados, redação e revisão crítica do artigo, LS Longarai trabalhou na coleta e organização de dados e na redação do artigo.

\section{Referências}

1. Hu FB. Dietary patterns analysis: a new direction in nutritional epidemiology. Curr Opin Lipidol 2002; 13(1):3-9.

2. Martins APB, Levy RB, Claro RM, Moubarac JC, Monteiro CA. Participação crescente de produtos ultraprocessados na dieta brasileira (1987-2009). Rev Saude Publica 2013; 47(4):656-665.

3. Costa Louzada ML, Martins AP, Canella DS, Baraldi LG, Levy RB, Claro RM, Moubarac JC, Cannon G, Monteiro CA. Ultra-processed foods and the nutritional dietary profile in Brazil. Rev Saude Publica 2015; 49:38.

4. Sichieri R, Castro JFG, Moura AS. Fatores associados ao padrão de consumo alimentar da população brasileira urbana. Cad Saude Publica 2003; 19(Suppl. 1):S47-S53.

5. Krieger JP, Pestoni G, Cabaset S, Brombach C, Sych J, Schader C, Faeh D, Rohrmann S. Dietary patterns and their sociodemographic and lifestyle determinants in Switzerland: results from the National Nutrition Survey menuCH. Nutrients 2018; 11(1):62.

6. Chen L, Zhu H, Gutin B, Dong Y. Race, gender, family structure, socioeconomic status, dietary patterns, and cardiovascular health in adolescents. Curr Dev Nutr 2019; 3(11):nzz117.

7. Pérez-Tepayo $S$, Rodríguez-Ramírez $S$, Unar-Munguía M, Shamah-Levy T. Trends in the dietary patterns of Mexican adults by sociodemographic characteristics. Nutr J 2020; 19:51.

8. Swinburn B, Sacks G, Vandevijvere S, Kumanyika S, Lobstein T, Neal B, Barquera S, Friel S, Hawkes C, Kelly B, L'abbé M, Lee A, Ma J, Macmullan J, Mohan S, Monteiro C, Rayner M, Sanders D, Snowdon W, Walker C; INFORMAS. INFORMAS (International Network for Food and Obesity/non-communicable diseases Research, Monitoring and Action Support): overview and key principles. Obes Rer 2013; 14(Suppl. 1):1-12.

9. Backes V. Ambiente alimentar urbano de São Leopoldo: identificação, descrição e relação com a obesidade [tese]. São Leopoldo: Universidade do Vale do Rio dos Sinos, Programa de Pós-Graduação em Saúde Coletiva; 2017.

10. Herforth A, Ahmed S. The food environment, its effects on dietary consumption, and potential for measurement within agriculture-nutrition interventions. Food Sec 2015; 7:505-520.

11. Azevedo SJS. Segregação e oportunidades de acesso aos serviços básicos de saúde em Campinas: vulnerabilidades sociodemográficas no espaço intra-urbano. Campinas: Unicamp, Núcleo de Estudos de População; 2014.

12. Instituto Brasileiro de Geografia e Estatística (IBGE). Censo Demográfico 2010. Características da população e dos domicílios: resultados do universo. Rio de Janeiro: IBGE; 2011.

13. Programa das Nações Unidas para o Desenvolvimento (PNUD), Instituto de Pesquisa Econômica e Aplicada IPEA, Fundação João Pinheiro. Atlas do Desenvolvimento Humano no Brasil. 2013. [acessado 2020 Mar 19]. Disponível em: http://www.atlasbrasil.org. br/2013 
14. Anjos LA, Wahrlich V, Vasconcellos MT, Souza DR Olinto MT, Waissmann W, Henn RL, Rossato SL, Lourenço AE, Bressan AW. Development of a food frequency questionnaire in a probabilistic sample of adults from Niterói, Rio de Janeiro, Brazil. Cad Saude Publica 2010; 26(11):2196-2204.

15. Machado FCS, Henn RL, Olinto MTA, Anjos LA, Wahrlich V, Waissmann W. Reprodutibilidade e validade de um questionário de frequência alimentar por grupos de alimentos, em adultos da Região Metropolitana de Porto Alegre, Brasil. Rev Nutr 2012; 25(1):65-77.

16. Panagiotakos D. -priori versus -posterior methods in dietary pattern analysis: a review in nutrition epidemiology. Nutrition Bulletin 2008; 33(4):311-315.

17. Olinto MTA. Padrões alimentares: análise de componentes principais. In: Kac G, Sichieri R, Gigante DP, organizadores. Epidemiologia nutricional. Rio de Janeiro: Fiocruz/Atheneu; 2007. p. 213-225.

18. Newby PK, Tucker KL. Empirically derived eating patterns using factor or cluster analysis: a review. Nutr Rev 2004; 62(5):177-203.

19. Hoffmann M, Mendes KG, Canuto R, Garcez AS, Theodoro H, Rodrigues AD, Dalpicolli A, Olinto MTA. Padrões alimentares de mulheres no climatério em atendimento ambulatorial no Sul do Brasil. Cien Saude Colet 2015; 20(5):1565-1574.

20. Hu FB, Rimm E, Smith-Warner SA, Feskanich D, Stampfer MJ, Ascherio A, Sampson L, Willett WC. Reproducibility and validity of dietary patterns assessed with a food-frequency questionnaire. Am J Clin Nutr 1999; 69(2):243-249.

21. Hair JF, Anderson RE, Tatham RL, Black WC. Análise de agrupamentos. In: Hair JF, Anderson RE, Tatham RL, Black WC. Análise multivariada de dados. Porto Alegre: Bookman; 2005. p. 380-419.

22. Lenz A, Olinto MTA, Dias-da-Costa JS, Alves AL, Balbinotti M, Pattussi MP, Bassani DG. Socioeconomic, demographic and lifestyle factors associated with dietary patterns of women living in Southern Brazil. Cad Saude Publica 2009; 25(6):1297-1306.

23. Alves ALS, Olinto MTA, Costa JSD, Bairros FS, Balbinotti MAA. Padrões alimentares de mulheres adultas residentes em área urbana no sul do Brasil. Rev Saude Publica 2006; 40(5): 865-873.

24. Ternus DL, Henn RL, Bairros F, Costa JS, Olinto MTA Padrões alimentares e sua associação com fatores sociodemográficos e comportamentais: Pesquisa Saúde da Mulher 2015, São Leopoldo (RS). Rev Bras Epidemiol 2019; 22:e190026.

25. Poltronieri TS, Gregoletto MLO, Cremonese C. Padrões alimentares e fatores associados em docentes de uma instituição privada de ensino superior. Cad Saude Colet 2019; 27(4):390-403.

26. Gimeno SGA, Mondini L, Moraes SA, Freitas ICM. Padrões de consumo de alimentos e fatores associados em adultos de Ribeirão Preto, São Paulo, Brasil: Projeto OBEDIARP. Cad Saude Publica 2011; 27(3):533545.

27. Ferreira-Nunes PM, Papini SJ, Corrente JE. Padrões alimentares e ingestão de nutrientes em idosos: análise com diferentes abordagens metodológicas. Cien Saude Colet 2018; 23(12):4085-4094.
28. Arruda SP, da Silva AA, Kac G, Goldani MZ, Bettiol $\mathrm{H}$, Barbieri MA. Socioeconomic and demographic factors are associated with dietary patterns in a cohort of young Brazilian adults. BMC Public Health 2014; 14:654.

29. Aguiar OB, Vasconcelos AGG, Barreiro PLD. Identificação de padrões alimentares: comparação das técnicas de análise de componentes principais e de principais eixos fatoriais. Rev Bras Epidemiol 2019; 22:e190048.

30. Cattafesta M, Zandonade E, Bissoli NS, Salaroli LB. Padrões alimentares de trabalhadores bancários e sua associação com fatores socioeconômicos, comportamentais e laborais. Cien Saude Colet 2019; 24(10):3909-3922.

31. Nogueira VC, Arruda SPM, Sampaio HAC, Rodrigues BC, Silva EB, Farias BO, Sabóia KM. Fatores socioeconômicos, demográficos e de estilo de vida associados a padrões alimentares de trabalhadores em turnos. Cien Saude Colet 2019; 24(3):761-769.

32. Cardoso Lde O, Carvalho MS, Cruz OG, Melere C, Luft VC, Molina Mdel C, Faria CP, Benseñor IM, Matos SM, Fonseca Mde J, Griep RH, Chor D. Eating patterns in the Brazilian Longitudinal Study of Adult Health (ELSA-Brasil): an exploratory analysis. Cad Saude Publica 2016; 32(5):e00066215.

33. Massarani FA, Cunha DB, Muraro AP, Souza BSN, Sichieri R, Yokoo EM. Agregação familiar e padrões alimentares na população brasileira. Cad Saude Publica 2015; 31(12):2535-2545

34. Olinto MTA, Willett WC, Gigante DP, Victora CG. Sociodemographic and lifestyle characteristics in relation to dietary patterns among young Brazilian adults. Public Health Nutr 2011; 14(1):150-159.

35. Assumpção D, Domene SMA, Fisberg RM, Canesqui AM, Barros MBA. Diferenças entre homens e mulheres na qualidade da dieta: estudo de base populacional em Campinas, São Paulo. Cien Saude Colet 2017; 22(2):347-358.

36. Lins APM, Sichieri R, Coutinho WF, Ramos EG, Peixoto MVM, Fonseca VM. Alimentação saudável, escolaridade e excesso de peso entre mulheres de baixa renda. Cien Saude Colet 2013; 18(2):357-366.

37. Figueiredo ICR, Jaime PC, Monteiro CA. Fatores associados ao consumo de frutas, legumes e verduras em adultos da cidade de São Paulo. Rev Saude Publica 2008; 42(5):777-785.

38. Baker AH, Wardle J. Sex differences in fruit and vegetable intake in older adults. Appetite 2003; 40(3):269-275.

39. Brasil. Ministério da Saúde (MS). Vigitel Brasil 2018 Vigilância de fatores de risco e proteção para doenças crônicas por inquérito telefônico. Brasília: MS; 2019.

40. Estima CCP, Philippi ST, Alvarenga MS. Fatores determinantes de consumo alimentar: por que os indivíduos comem o que comem? Rev Bras Nutr Clin 2009; 24(4):263-268.

41. Avelar AE, Rezende DC. Hábitos alimentares fora do lar: um estudo de caso em Lavras-MG. Organizações Rurais \& Agroindustriais 2013; 15(1):137-152.

42. Bielemann RM, Motta JVS, Minten GC, Horta BL, Gigante DP. Consumo de alimentos ultraprocessados e impacto na dieta de adultos jovens. Rev Saude Publica 2015; 49:28. 
43. D’Avila HF, Kirsten VR. Consumo energético proveniente de alimentos ultraprocessados por adolescentes. Rev Paul Pediatr 2017; 35(1):54-60.

44. Enes CC, Camargo CM, Justino MIC. Ultra-processed food consumption and obesity in adolescents. Rev Nutr 2019; 32:e180170.

45. Moubarac JC, Batal M, Louzada ML, Martinez Steele E, Monteiro CA. Consumption of ultra-processed foods predicts diet quality in Canada. Appetite 2017; 108:512-520.

46. Martinez Steele E, Baraldi LG, Louzada MLC, Moubarac JC, Mozaffarian D, Monteiro CA. Ultra-processed foods and added sugars in the US diet: evidence from a nationally representative cross-sectionalstudy. $B M$ J Open 2016; 6(3):e009892.

47. Monteiro CA, Moubarac JC, Levy RB, Canella DS, Louzana MLC, Cannon G. Household availability of ultra-processed foods and obesity in nineteen European countries. Public Health Nutr 2018; 21(1):18-26.

48. Judd SE, Gutiérrez OM, Newby PK, Howard G, Howard VJ, Locher JL, Kissela BM, Shikany JM. Dietary patterns are associated with incident stroke and contribute to excess risk of stroke in Black Americans. Stroke 2013; 44(12):3305-3311.

49. Canuto R, Fanton M, Lira PIC. Iniquidades sociais no consumo alimentar no Brasil: uma revisão crítica dos inquéritos nacionais. Cien Saude Colet 2019; 24(9):3193-3212.

50. Filomena S, Scanlin K, Morland KB. Brooklyn, New York foodscape 2007-2011: a five-year analysis of stability in food retail environments. Int J Behav Nutr Phys Act 2013; 10:46.

51. Mook K, Laraia BA, Oddo VM, Jones-Smith JC. Food security status and barriers to fruit and vegetable consumption in two economically deprived communities of Oakland, California, 2013-2014. Prev Chronic Dis 2016; 13:E21.

52. Boyle M, Stone-Francisco, S, Samuels SE. Environmental strategies and policies to suppor the althy eating and physical activity in low-income communities. Journal of Hunger \& Environmental Nutrition 2007; 1(2): 3-25.

53. Story M, Nanney MS, Schwartz MB. Schools and obesity prevention: creating school environments and policies to promote healthy eating and physical activity. Milbank Q 2009; 87(1):71-100.
54. Stern D, Ng SW, Popkin BM. The nutrient content of U.S. household food purchases by storetype. Am J Prev Med 2016; 50(2):180-190.

55. Taillie LS, Ng SW, Popkin BM. Global growth of "big box" stores and the potential impact on human health and nutrition. Nutr Rev 2016; 74(2):83-97.

56. Queiroz PWV, Coelho AB. Alimentação fora de casa: uma investigação sobre os determinantes da decisão de consumo dos domicílios brasileiros. Análise Econômica 2017; 35:67-104.

57. Andrade GC. Consumo de alimentos ultraprocessados fora de domicílio no Brasil [dissertação]. São Paulo: Universidade de São Paulo, Faculdade de Medicina; 2017.

58. Bezerra IN, Cavalcante JB, Moreira TMV, Mota CC, Sicheiri R. Alimentação fora de casa e excesso de peso: uma análise dos mecanismos explicativos. Rev Bras Promoç Saude 2016; 29(3):455-461.

59. Instituto Brasileiro de Geografia e Estatística (IBGE). Pesquisa de orçamentos familiares 2017-2018: primeiros resultados. Rio de Janeiro: IBGE; 2019.

60. Bezerra IN, Sichieri R. Características e gastos com alimentação fora do domicílio no Brasil. Rev Saude Publica 2010; 44(2):221-229.

61. World Health Organization (WHO). Diet, nutrition and prevention of chronic diseases. Geneva: WHO; 2003.

Artigo apresentado em 22/05/2020

Aprovado em 13/01/2021

Versão final apresentada em 15/01/2021

Editores-chefes: Romeu Gomes, Antônio Augusto Moura da Silva 